\title{
A Giant Occipital Encephalocele
}

\author{
Ashfaq Ul Hassan \\ Anatomy SKIMS Medical College Bemina \\ Corresponding Author: ashhassan@rediffmail.com
}

Copyright (C 2013 Horizon Research Publishing All rights reserved.

\begin{abstract}
Encephaloceles are rare Lesions. These defects in the formation of the cranium are often associated with congenital anomalies of the brain and/or meninges. The defect is often in the squamous part of the occipital bone and may include the posterior part of the foramen magnum. When the defect is small, usually only the meninges herniate and the anomaly is a cranial meningocele, or cranium bifidum with meningocele. Here we present a case of a six month baby who presented with progressive swelling in Occipital region.
\end{abstract}

Keywords Encephalocele, Optic Nerve, Herniation, Bands, Brain, Chiari

\section{Introduction}

Encephaloceles are rare Lesions These defects in the formation of the cranium are often associated with congenital anomalies of the brain and/or meninges. The defect is often in the squamous part of the occipital bone and may include the posterior part of the foramen magnum. When the defect is small, usually only the meninges herniate and the anomaly is a cranial meningocele, or cranium bifidum with meningocele. Here we present a case of a six month baby who presented with progressive swelling in Occipital region.

\section{Methodology and Discussion}

It is a form of neural tube defect caused by abnormal development of the neural tube. Cranium bifidum associated with herniation of the brain and/or its meninges occurs in rare circumstances. When the cranial defect is large, the meninges and part of the brain herniate, forming a meningoencephalocele. If the protruding brain contains part of the ventricular system, the anomaly is a meningohydroencephalocele.

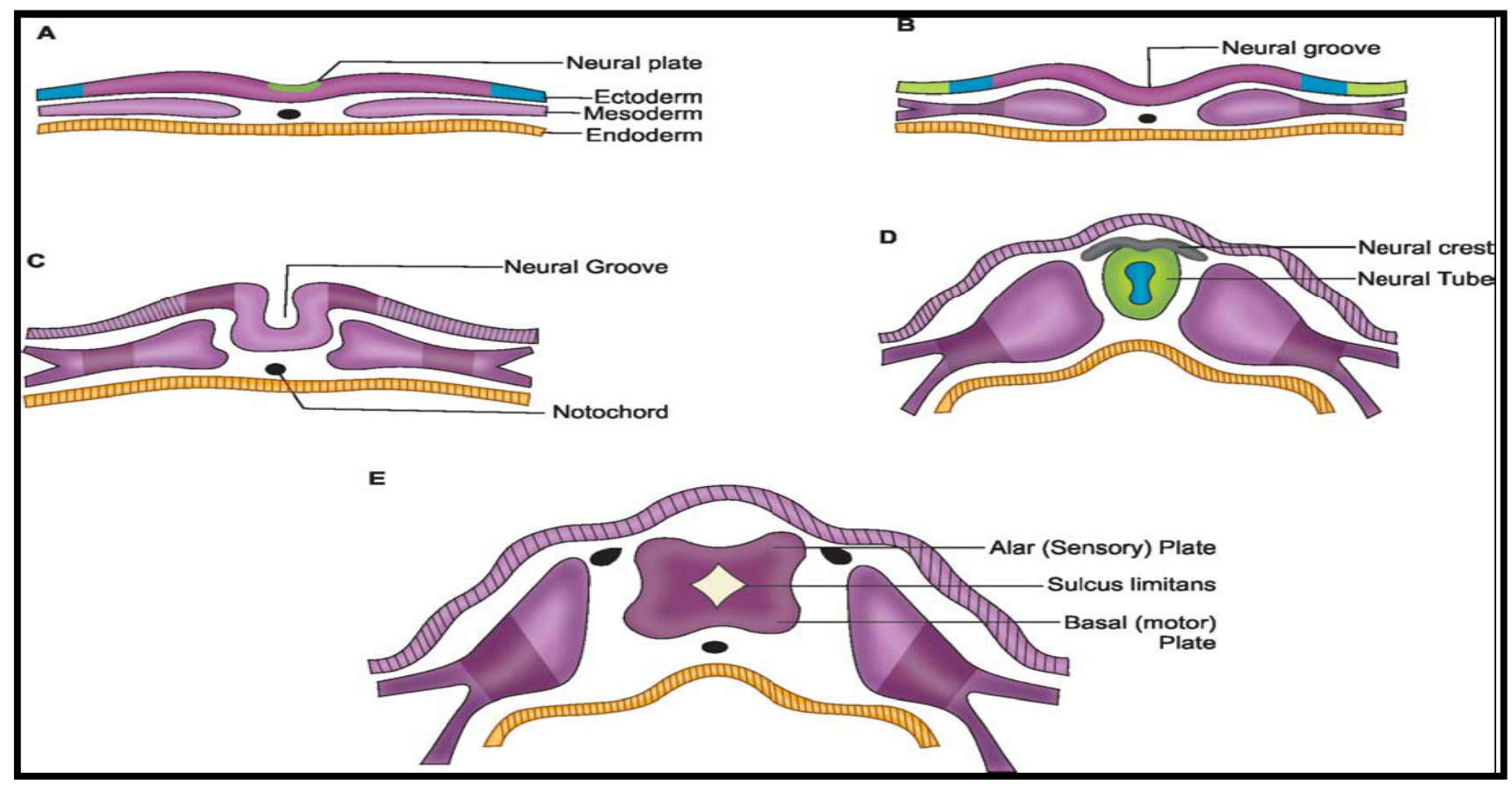

Figure 1. Development of Neural tube 


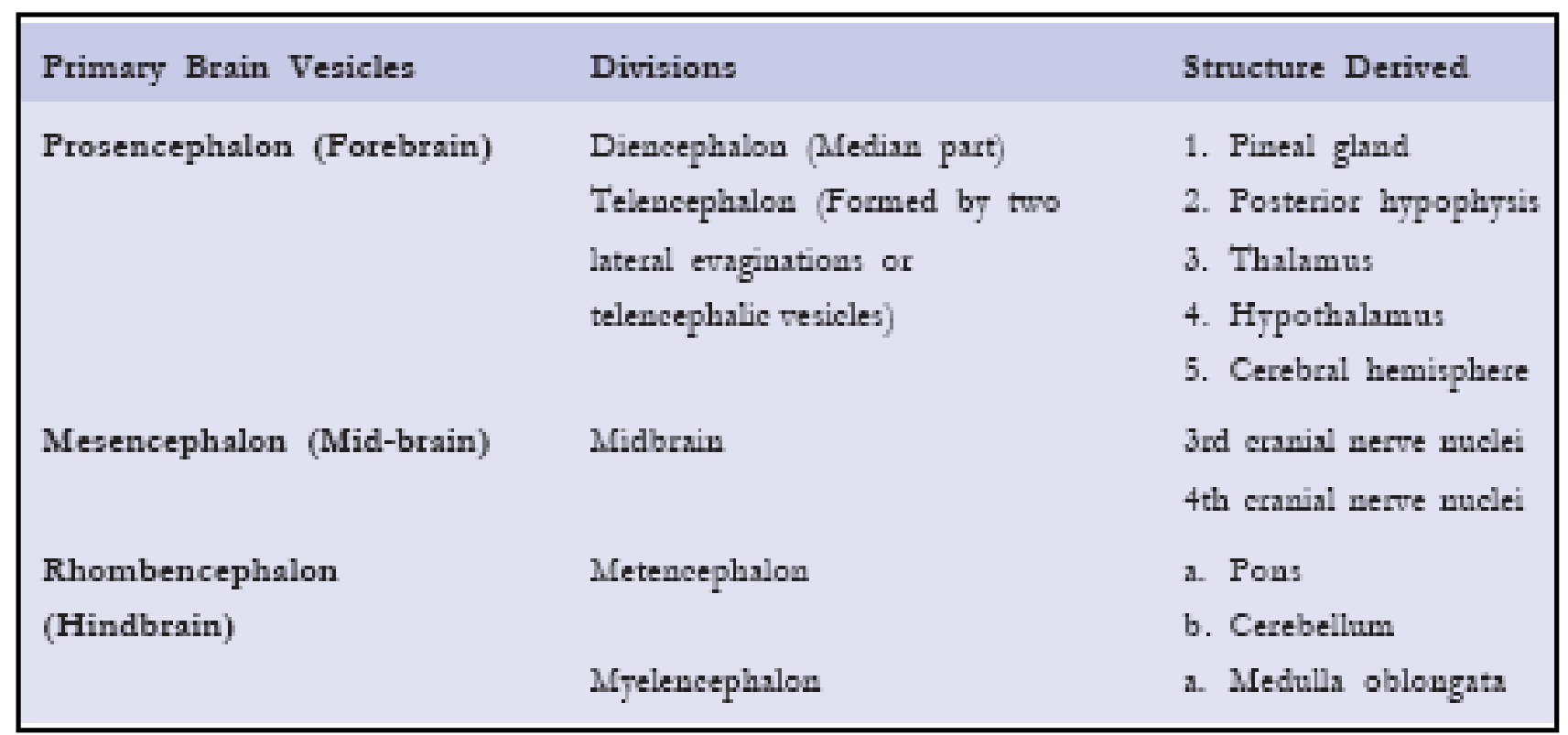

Figure 2. Development of Brain . Tabulated

The incidence of encephalocele varies according to geographic region. The location of the encephalocele also exhibits geographic variability, with frontonasal encephaloceles more common in Southeast Asia and occipital encephaloceles more common in North America. The degree of herniation of neural tissue into the encephalocele sac may be highly variable. MRI scanning serves to classify the type of encephalocele, as well as to determine the presence of neural tissues within the sac. The treatment of encephaloceles is surgical resection of the lesion at its base, repair of the dura, and bone grafting to cover the calvarial defect. In some occipital encephaloceles, the sac may contain vital neural or vascular structures, and such repair is not possible. The outcome of surgical treatment depends on the location of the encephalocele and the amount of neural tissue remaining within the cranial vault. In general, occipital encephaloceles are more commonly associated with hydrocephalus and a worse cognitive outcome ${ }^{[1]}$

Encephaloceles and meningoceles may present in the nasal cavity through defects in the cribriform plate. Clinically they have an appearance similar to that of a nasal polyp.

A cranial meningocele consists of a CSF-filled meningeal sac only, and a cranial encephalocele contains the sac plus cerebral cortex, cerebellum, or portions of the brain stem. Microscopic examination of the neural tissue within an encephalocele is often abnormal. The cranial defect occurs most commonly in the occipital region at or below the inion, but in certain parts of the world frontal or nasofrontal encephaloceles are more prominent. ${ }^{[2]}$

Infants with a cranial encephalocele are at increased risk for developing hydrocephalus due to aqueduct stenosis, a Chiari malformation, or the Dandy-Walker syndrome. Examination may show a small sac with a pedunculated stalk or a large cyst-like structure that may exceed the size of the cranium. The lesion may be completely covered with skin, but areas of denuded skin can occur and require urgent surgical management. Transillumination of the sac may indicate the presence of neural tissue. A plain roentgenogram of the skull and cervical spine is indicated to define the anatomy of the vertebra. An ultrasound is most helpful in determining the contents of the sac, obviating the need for a $\mathrm{CT}$ scan in most cases. Children with a cranial meningocele generally have a good prognosis, whereas patients with an encephalocele are at risk for visual problems, microcephaly, mental retardation, and seizures. Generally, children with neural tissue within the sac and associated hydrocephalus have the poorest prognosis. Giant Meningoceles posses a surgical challenge $[3,4.5]$

\section{Associations of Encephalocele}

Meckel-Gruber syndrome is a rare autosomal recessive condition that is characterized by an occipital encephalocele, cleft lip or palate, microcephaly, microphthalmia, abnormal genitalia, polycystic kidneys, and polydactyly.

Congenital anomalies of the posterior fossa, including the Dandy-Walker syndrome, the Chiari malformation, and encephalocele, are prominently associated with ataxia because of their destruction or replacement of the cerebellum.

Optic nerve hypoplasia may occur alone or with other developmental abnormalities, including microphthalmia, anencephaly, hydrocephalus, and encephalocele.

Morning glory disk anomaly: is a congenital malformation of the optic nerve characterized by an enlarged, excavated, funnel-shaped disk with an elevated rim, resembling the flower for which it is named. The anomaly may be associated with developmental midline defects, including cleft lip and palate, agenesis of the corpus callosum, hypertelorism, and 
encephalocele.

The limb-body wall complex (LBWC) involves vascular disruption early in development, affecting several embryonic structures, including at least two of the following three characteristics: exencephaly or encephalocele with facial clefts, thoraco- and/or abdominoschisis, and limb defects.

Adhesive bands involve the craniofacial area and are associated with severe defects such as encephalocele and facial clefts. Adhesive bands result from broad fusion between disrupted fetal parts and an intact amniotic membrane. The craniofacial defects do not appear to be caused by constrictive amniotic bands but result from a vascular disruption sequence with or without cephaloamniotic adhesion.

Other Associations are Neurofibromatosis 1, Meckel-Gruber Syndrome, von Voss syndrome, Chemke syndrome, Roberts syndrome, and Knobloch syndromes , Microcephaly and Micrognathia ${ }^{[6]}$

Factors which determine the prognosis of patients diagnosed with occipital Encephalocele include the size of the sac, the contents of the neural tissue, hydrocephaly, infections, and pathologies that accompany the condition. An occipital Encephalocele is a congenital neurologic condition with an extremely high morbidity and mortality in spite of the treatments rendered pre- and postoperatively ${ }^{[7]}$

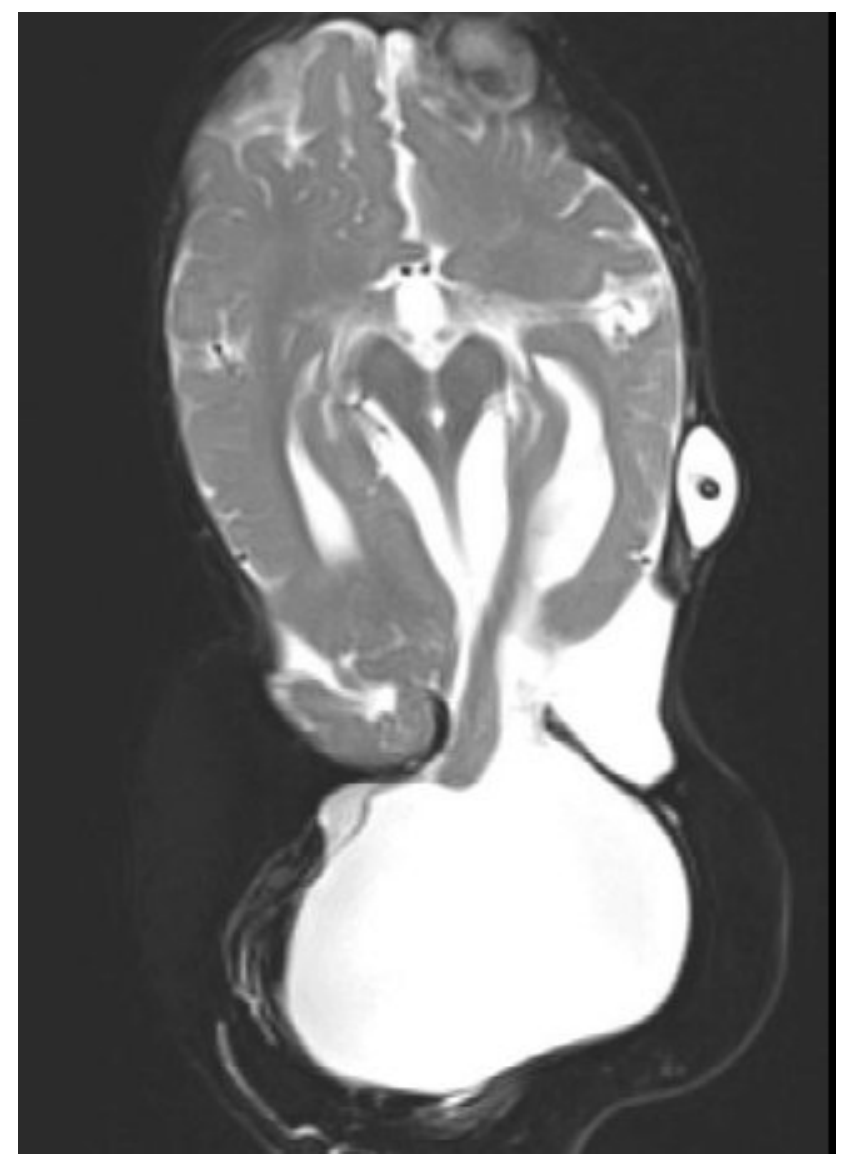

Figure 3. CT Scan of Brain showing Giant Encephalocele

\section{Conclusion}

Encephaloceles are rare and complicated defects. Survival is not great. They are often associated with congenital anomalies of the brain and/or meninges. A thorough look out for such cases is warranted in case of a gradually increasing swelling in the head region. The article represents a case of a Giant Encephalocele in the occipital region with a brief review of literature and the possible rare associations which can be associated with it and effect the survival of the patients.

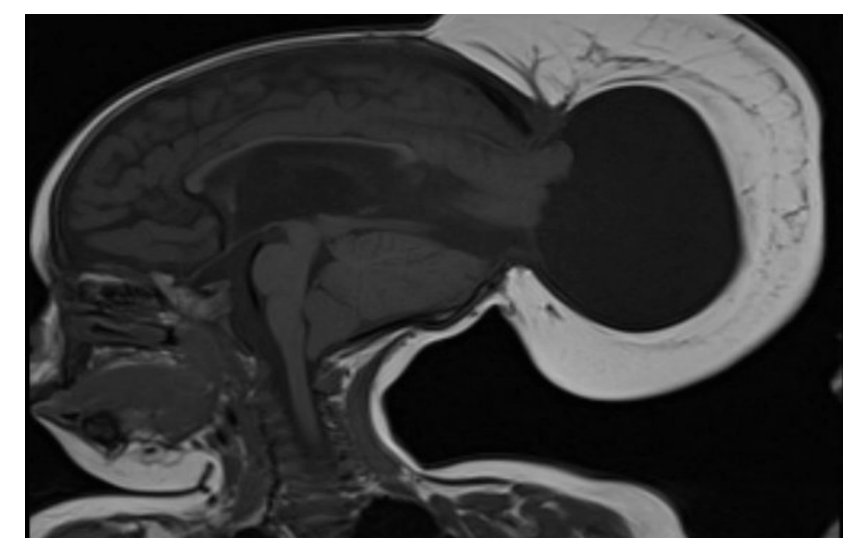

Figure 4. CT Scan of Brain showing Giant Occipital Encephalocele

\section{REFERENCES}

[1] Morón FE, Morriss MC, Jones JJ et-al. Lumps and bumps on the head in children: use of CT and MR imaging in solving the clinical diagnostic dilemma. Radiographics.

[2] Hoving EW: Nasal encephaloceles. Childs Nerv Syst 2000;16:702-706.

[3] Agrawal D, Mahapatra AK. Giant occipital encephalocele with microcephaly and micrognathia. Pediatr Neurosurg 2004;40:205-6

[4] Ozlu O, Sorar M, Sezer E, Bayraktar N. Anesthetic management in two infants with giant occipital encephalocele. Paediatr Anaesth 2008;18:792-3.

[5] Bayramicli M, Sonmez A, Yavas T. Microvascular reconstruction of a giant encephalocele defect in a 10-week-old infant. Br J Plast Surg 2004;57:89-92.

[6] Agrawal D, Mahapatra AK: Giant occipital encephalocele with microcephaly and micrognathia. Pediatr Neurosurg 2004;40:205-206.

[7] Prognostic factors in patients with occipital encephalocele. Kiymaz N, Yilmaz N, Demir I, Keskin S. Pediatr Neurosurg. 2010;46(1):6-11. doi: 10.1159/000314051. 